www.nature.com/pj

\title{
Development of an antibacterial chitin betainate wound dressing
}

\author{
Wanpen Tachaboonyakiat ${ }^{1,2}$, Ekkachai Sukpaiboon ${ }^{1}$ and Onruthai Pinyakong ${ }^{3}$
}

This research involved the chemical modification of chitin with positively charged groups to confer antibacterial activity. Chitin betainate, a quaternary ammonium chitin, was prepared by the acylation of chitin with carboxymethyl trimethyl ammonium chloride. The chemical structure was elucidated by Fourier transform infrared spectroscopy (FTIR), which revealed the presence of the additional ester peak at $1738 \mathrm{~cm}^{-1}$ and some change in amide I at $1650 \mathrm{~cm}^{-1}$. Chitin and chitin betainate gels were prepared and then tested for their water absorption, contact angle and antibacterial activity. The chitin betainate had a 1.07-fold higher water-absorption level and a higher hydrophilic property than chitin, and it also exhibited an antibacterial activity against Staphylococcus aureus and Escherichia coli. At a final concentration of $10 \mathrm{mg} \mathrm{ml}^{-1}$, chitin betainate completely killed E. coli within $10 \mathrm{~min}$, and $S$. aureus was reduced by $45.2 \%$ and $78 \%$ after $10 \mathrm{~min}$ and $24 \mathrm{~h}$, respectively. The antibacterial activity against $S$. aureus was dose-dependent, whereas no antibacterial activity was observed against $E$. coli when using chitin betainate at $<10 \mathrm{mg} \mathrm{ml}^{-1}$. The addition of PEG4000 to either chitin or chitin betainate further increased the water absorption by 2.0 - and 2.5 -fold, respectively. Therefore, chitin betainate is expected to be suitable as an antibacterial wound-dressing material.

Polymer Journal (2014) 46, 505-510; doi:10.1038/pj.2014.47; published online 18 June 2014

Keywords: antibacterial; betainate; chitin; quaternary ammonium; wound dressing

\section{INTRODUCTION}

Chitin, 2-acetamido-2-deoxy-(1-4)- $\beta$-D-glucopyranose, is a naturally occurring and abundant polysaccharide that is found in the cell walls of fungi and the exoskeletons of arthropods and crustaceans, including in the shells of crustaceans. Chitin has several desirable biological properties, including its high biocompatibility, biodegradability and excellent wound-healing property. ${ }^{1,2}$ Interestingly, it has been found to induce granulation tissue with angiogenesis and lead to less scar tissue formation ${ }^{3-6}$ as well as provide pain relief. ${ }^{7}$ In addition, chitin can be degraded by lysozyme in the human body and thus does not pose a long-term foreign-body risk if included within the wound. These traits of chitin make it of interest in biomedical applications, especially for use as a biodegradable wound dressing. Because a wound dressing must have direct contact with the (infected) wound site, antibacterial and water-absorption properties are advantageous for any wound-dressing material. However, chitin itself exhibits no significant antibacterial activity, in contrast to chitosan, 2-amino-2-deoxy-(1-4)- $\beta$-D-glucopyranose, a deacetylated copolymer of chitin that contains amino groups instead of acetamido groups. The presence of the amino groups in chitosan enables distinctive biological functions, such as antibacterial and hemostatic activities. ${ }^{8}$ Accordingly, much attention has been paid to chitosan and its positive charged derivatives for antimicrobial applications. ${ }^{9-13}$ By contrast, there is very little published information about the antimicrobial activity of chitin derivatives, covering only aminoethylchitin $^{14}$ and chitooligosaccharide-glycidyltrimethyl ammonium chloride. ${ }^{15}$ However, the aminoethyl groups were protonated only in an acidic condition, which might be the major drawback in terms of eliciting an antimicrobial activity at the more physiologically relevant near-neutral $\mathrm{pH}$ of plasma and dermal tissues. Therefore, we were interested in modifying chitin to have permanent positive charges at a near-neutral $\mathrm{pH}$ range. In addition, chitin can be conveniently gelled by solvent exchange, indicating that the obtained modified chitin gels are potentially suitable as antibacterial wound dressings.

Quaternary ammonium compounds are organic compounds that contain at least one long hydrophobic alkyl chain attached to a positively charged central nitrogen atom. These compounds are widely used as antimicrobial agents because of their biocidal activity. Betaine was selected as the quaternary ammonium compound for this research, because like chitin, it is a natural and non-toxic product, and it is abundant in sugar beet.

In the present work, chitin was modified with betainate via acylation (ester/amide) in order to substitute the permanent positive

${ }^{1}$ Department of Materials Science, Faculty of Science, Chulalongkorn University, Bangkok, Thailand; ${ }^{2}$ Unit Cell for Research and Development of Herbals and Natural Products for Dental Application, Faculty of Dentistry, Chulalongkorn University, Bangkok, Thailand and ${ }^{3}$ Department of Microbiology, Faculty of Science, Chulalongkorn University, Bangkok, Thailand

Correspondence: Dr W Tachaboonyakiat, Department of Materials Science, Chulalongkorn University, Phayathai Road, Pathumwan, Bangkok 10330, Thailand. E-mail: Wanpen.Ta@chula.ac.th

Received 28 February 2014; revised 15 April 2014; accepted 17 April 2014; published online 18 June 2014 
charges of quaternary ammonium groups. Linking the cationic substitute to the chitin backbone via an ester bond would likely result in a more biodegradable product than that obtained via an ether bond. The use of amide bonds would also result in a biodegradable product. Chitin and chitin betainate could be conveniently processed into a gel and shaped by simple solvent exchange. To evaluate the potential utility of chitin betainate as antibacterial wound dressing, we also investigated its antibacterial activity and water-absorbance levels.

\section{EXPERIMENTAL PROCEDURE}

\section{Materials}

Chitin with a degree of acetylation (DA) of 62, as determined by Fourier transform infrared spectroscopy (FTIR), was purchased from Sigma Aldrich (Munich, Germany). Carboxyl methyl trimethyl ammonium chloride (CTA betaine hydrochloride) and dicyclohexyl carbodiimide (DCC) were purchased from Fluka (Munich, Germany). Polyethylene glycol (PEG) with a molecular weight $\left(\mathrm{M}_{\mathrm{W}}\right)$ of 4000 (PEG4000) was purchased from Asia Pacific Specialty Chemicals Limited (Seven Hills, New South Wales, Australia). Dimethyl acetamide (DMAc) and acetone were obtained from RCI Lab Scan Ltd. (Samutsakorn, Thailand). Lithium chloride ( $\mathrm{LiCl})$ was purchased from Ajax Finechem Co., Ltd. (Auckland, New Zealand). Sodium dihydrogen phosphate dehydrate and disodium hydrogen phosphate dodecahydrate were purchased from Merck Co., Ltd. (Darmstadt, Germany). Staphylococcus aureus (ATCC 6538) and Escherichia coli (ATCC 25923) were gifted from the Department of Microbiology, Faculty of Science, Chulalongkorn University, Bangkok, Thailand. Difco Luria-Bertani (LB) and Miller nutrient were supplied by Becton, Dickinson and Co. (Franklin Lakes, NJ, USA).

\section{Purification of chitin and its modification to chitin betainate}

Chitin was purified before chemical modification. Chitin was dissolved in DMAc/5\% (w/v) LiCl to a final concentration of $0.5 \%(\mathrm{w} / \mathrm{v})$. The solution was centrifuged to remove the insoluble impurities, and then the chitin was precipitated from the supernatant in a large amount of distilled water and then washed several times with acetone and dried under vacuum.

Chitin (2 g; $0.01 \mathrm{~mole}$ ) was dissolved in $150 \mathrm{ml}$ of DMAc/5\% (w/v) LiCl, and then CTA ( $3.07 \mathrm{~g} ; 0.02$ mole) was added and stirred until completely dissolved. The acylation of chitin with CTA was performed using DCC ( $8.24 \mathrm{~g} ; 0.04$ mole) as the coupling agent at room temperature $\left(\sim 25-28^{\circ} \mathrm{C}\right)$ for $24 \mathrm{~h}$. The mixture was then centrifuged to remove the $N, N^{\prime}$-dicyclohexylurea (DCUrea) byproduct. Next, the chitin betainate was precipitated from the supernatant in acetone, washed with acetone several times and dried under vacuum.

\section{Structural characterization of chitin betainate and its degree of substitution (DS)}

The chemical modification of chitin with CTA was confirmed by FTIR analysis (Nicolet 6700, Thermo Scientific, Madison, WI, USA). The obtained chitin betainate was ground with potassium bromide $(\mathrm{KBr})$ into a pellet. The infrared absorption was scanned in the range of $4000-400 \mathrm{~cm}^{-1}$ for 64 scans at a resolution of $4.0 \mathrm{~cm}^{-1}$.

The DS of betainate was estimated from the degree of acylation, calculated from the integral ratios between the carbonyl groups of the ester/amide I and $\mathrm{C}-\mathrm{O}$ of the pyranose rings from Equation (1);

$$
\text { DS (acylation })=\left(A_{1735}+A_{1650}\right) / A_{1072}
$$

where $A_{1735}, A_{1650}$ and $A_{1072}$ are the integral areas under the peak of the ester group at $1735 \mathrm{~cm}^{-1}$, the amide I group at $1650 \mathrm{~cm}^{-1}$ and the $\mathrm{C}-\mathrm{O}$ of pyranose ring at $1072 \mathrm{~cm}^{-1}$, respectively. However, the final DS should be subtracted from the original chitin.

\section{Bactericidal activity}

The antibacterial activity of each sample was evaluated against $S$. aureus as a representative Gram-positive bacterium and E. coli as a representative Gramnegative bacterium. Antibacterial activities were determined in terms of the required time and concentration for bactericidal activity as follows.
For the evaluation of the bactericidal activity with increasing exposure time, S. aureus and E. coli were cultured in LB nutrient broth $(5 \mathrm{ml})$ at $30^{\circ} \mathrm{C}$ for $24 \mathrm{~h}$. The suspensions of bacteria were centrifuged and washed with phosphate buffer and adjusted to a final bacterial concentration of $3.5 \times 10^{6}$ colonyforming units $(\mathrm{CFU}) \mathrm{ml}^{-1}$. Chitin and chitin betainate were sterilized with ultraviolet irradiation for $30 \mathrm{~min}$ and then added to bacterial suspensions at $10 \mathrm{mg} \mathrm{m}^{-1}$ and incubated for the desired time (10,20, 40 and $60 \mathrm{~min}$ and $24 \mathrm{~h}$ ) with shaking. After the incubation, the total number of viable bacteria $\left(\mathrm{CFU} \mathrm{ml}{ }^{-1}\right)$ in the bacterial suspension was determined using the total plate count method. To this end, each bacterial suspension was serial diluted, and $100 \mu \mathrm{l}$ aliquots were plated per 9-cm-diameter LB agar plate followed by incubation at $30^{\circ} \mathrm{C}$ for $24 \mathrm{~h}$. The viable bacterial colonies were then counted. The percentage of reduction in the viable cell number was determined from Equation (2) from triplicate experiments,

$\%$ reduction $=\frac{\log \left(\text { nunber of bacteri } a_{\text {before }}\right)-\log \left(\text { number of bacteria }_{\text {after }}\right)}{\log \left(\text { number of bacteria }_{\text {before }}\right)} \times 100$

To evaluate the concentration-dependent bactericidal activity, the suspensions of bacteria were prepared as described above at a final concentration of $3.5 \times 10^{6} \mathrm{CFU} \mathrm{ml}^{-1}$. Chitin and chitin betainate were sterilized as above. To determine the minimal concentration for bacterial killing, bacteria were incubated with the chitin or chitin betainate at final concentrations of 10 , 5 and $1 \mathrm{mg} \mathrm{ml}^{-1}$ with shaking for the appropriate time for bactericidal activity (obtained from the above evaluation). The bacterial suspensions were then evaluated for the total viable cell number and the percentage of viable cell reduction, as above.

\section{Preparation of chitin and chitin betainate hydrogels}

Chitin or chitin betainate was dissolved in DMAc/5\% (w/v) LiCl to a final concentration of $0.5 \%(\mathrm{w} / \mathrm{v})$. Next, 2-ml aliquots of the chitin or chitin betainate solution were placed into cubic molds $\left(1.5 \times 1.5 \times 1.5 \mathrm{~cm}^{3}\right)$, stabilized in shape by immersing in acetone at room temperature for $24 \mathrm{~h}$ and then dried under vacuum to yield the chitin or chitin betainate gel.

To increase the water absorbability of the chitin or chitin betainate gels, $0.1 \%(\mathrm{w} / \mathrm{v})$ PEG4000 was mixed homogeneously with the $0.5 \%(\mathrm{w} / \mathrm{v})$ chitin or chitin betainate solution to form semi-interpenetrating networks. Next, 2-ml aliquots of the chitin/PEG4000 or chitin betainate/PEG4000 solutions were molded to form gels as described above.

\section{Water absorption}

The chitin, chitin betainate, chitin/PEG4000 and chitin betainate/PEG4000 gels were cut into $5 \times 5 \times 1 \mathrm{~mm}^{3}$ cubes, and the dry weight $\left(\mathrm{W}_{\mathrm{d}}\right)$ of each gel was determined. The gels were then swollen in $10 \mathrm{ml}$ of distilled water for $48 \mathrm{~h}$, and the wet weight $\left(\mathrm{W}_{\mathrm{w}}\right)$ of each gel was evaluated. The percentage of water absorption was then calculated from Equation (3),

$$
\% \text { Water absorption }=\left(W_{w}-W_{d}\right) / W_{d} \times 100
$$

The experiment was performed in triplicate, and the percentage of waterabsorption data are presented as the mean \pm s.d.\%.

\section{Hydrophilic-hydrophobic property}

The hydrophilic or hydrophobic property of the chitin, chitin betainate, chitin/PEG4000 and chitin betainate/PEG4000 gels were evaluated using a contact-angle meter (CAM-PLUS Tantec, Glendale Heights, IL, USA). The experiments were performed five times for each sample.

\section{Crystallinity}

X-ray diffraction of the samples was measured using a Bruker AXS Model D8 (Bruker, Madison, WI, USA). The X-ray source originated from $\mathrm{CuK} \alpha$ $(\lambda=0.154 \mathrm{~nm})$. The chitin, chitin betainate, chitin/PEG4000 and chitin betainate/PEG4000 gels were scanned at $2 \theta$ in the range of $15-55^{\circ}$ at a scan speed of $0.02^{\circ} \mathrm{s}^{-1}$. 
<smiles>C[N+](C)(C)CC(=O)O</smiles>

Carboxymethyl trimethyl ammonium chloride (CTA)<smiles>C(=NC1CCCCC1)C1CCCCC1</smiles>

Dicyclohexyl carbodiimide (DCC)<smiles>CCOCCOCCO</smiles>

$$
\downarrow
$$

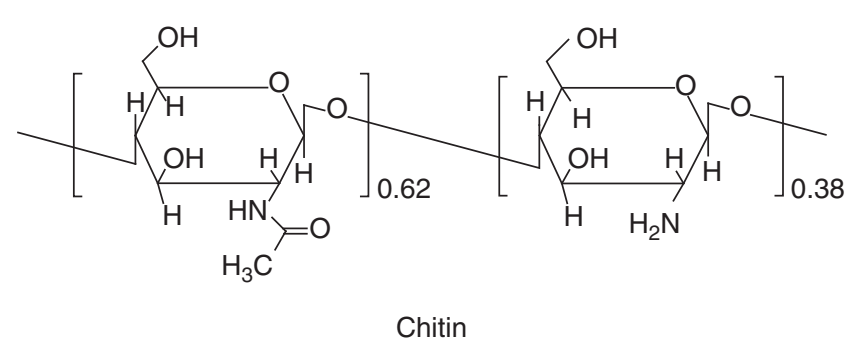

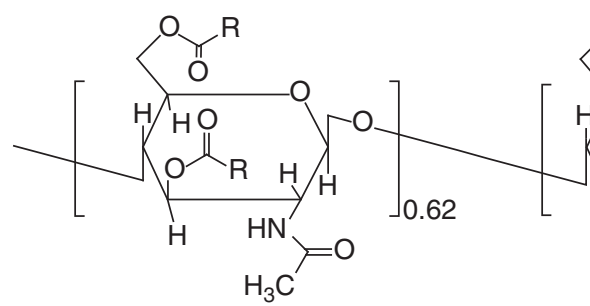

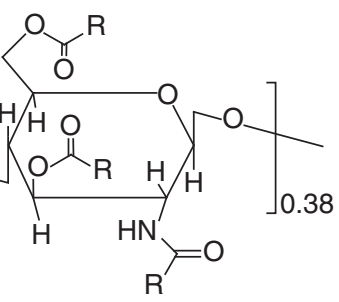<smiles>O=C(NC1CCCCC1)NC1CCCCC1</smiles><smiles>[R]=C[N+](C)(C)C</smiles>

Chitin betainate

Scheme 1 Acylation of chitin with CTA using DCC as the coupling agent.

\section{RESULTS AND DISCUSSION}

Chitin, a polymer that is naturally occurring and essentially non-toxic to humans, owing to its ability to be degraded by lysozyme in human body, has attracted interest because of its potential use in biomedical applications, especially for biodegradable wound dressings. The ideal wound dressing should have (i) good water absorbability, in order to absorb the exudate from the wound, and (ii) an antibacterial property, in order to protect the wound from infection. However, chitin does not have any functional groups that confer an antibacterial property. By contrast, chitosan has amino groups that can be protonated to $\mathrm{NH}_{3}{ }^{+}$in acidic conditions and exhibits antibacterial activity; however, this activity is markedly reduced at the physiological near-neutral $\mathrm{pH}$ of the plasma and skin tissue exudates.

It was our aim to chemically modify chitin with positive charges using the quaternary ammonium salt CTA to yield chitin betainate, with the expectation that it would induce an antibacterial property at near-neutral pH. Another attractive motivation for using (modified) chitin as a wound dressing is that a (modified) chitin gel can be prepared easily by simply replacing the solvent-dissolving chitin (DMAc/5\% (w/v) LiCl) with poor chitin solvents, such as acetone. The (modified) chitin gels can easily be shaped with a small amount of moisture and acetone. However, chitin itself possesses low hydrophilicity and so would not absorb much wound exudates (plasma). To prepare a good water-absorption wound dressing, the water absorbability of the hydrogel was enhanced in this work by the formation of a semi-interpenetrating network with the hydrophilic polymer PEG4000, because the availability of the terminal hydroxyl groups of PEG can improve the hydrophilicity as well as the water absorption of materials.

\section{Chemical synthesis of chitin betainate}

Chitin betainate was successfully synthesized via the acylation of the hydroxyl groups of chitin and/or amino groups in partly deacetylated chitin with the carboxylic groups of CTA by using DCC as the 


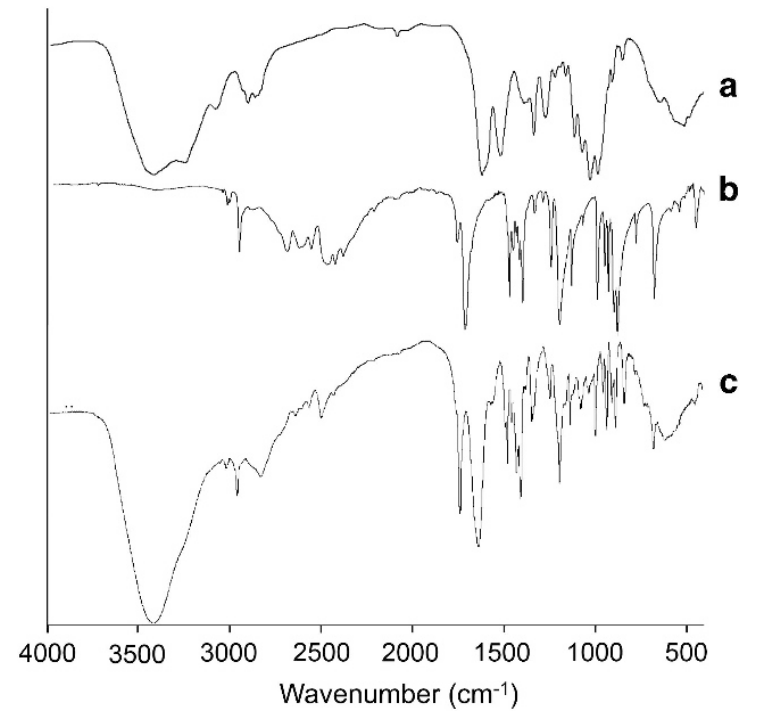

Figure 1 FTIR spectra of (a) chitin, (b) CTA and (c) chitin betainate.

Table 1 The number of viable cells and percentage of reduction of the viable cell number of Escherichia coli and Staphylococcus aureus after different exposure times to chitin or chitin betainate at $10 \mathrm{mg} \mathrm{ml}^{-1}$ compared with the control

\begin{tabular}{|c|c|c|c|c|}
\hline Microorganism & Sample & $\begin{array}{l}\text { Time } \\
\text { (min) }\end{array}$ & $\begin{array}{c}\text { Viable bacteria } \\
\qquad\left(\mathrm{CF} \cup \mathrm{ml}^{-1}\right)\end{array}$ & \% Reduction \\
\hline \multirow[t]{9}{*}{ E. coli } & Blank & 10 & $3.0 \times 10^{6} \pm 0.0$ & - \\
\hline & & 20 & $3.0 \times 10^{6} \pm 0.0$ & - \\
\hline & & 60 & $3.6 \times 10^{6} \pm 0.1$ & - \\
\hline & Chitin (10 $\mathrm{mg} \mathrm{ml}^{-1}$ ) & 10 & $3.1 \times 10^{6} \pm 0.1$ & 0 \\
\hline & & 20 & $2.7 \times 10^{6} \pm 0.3$ & 0.6 \\
\hline & & 60 & $1.8 \times 10^{6} \pm 1.1$ & 4.6 \\
\hline & Chitin betainate & 10 & $0 \pm 0.0$ & 100.0 \\
\hline & $\left(10 \mathrm{mg} \mathrm{ml}^{-1}\right)$ & 20 & $0 \pm 0.0$ & 100.0 \\
\hline & & 60 & $0 \pm 0.0$ & 100.0 \\
\hline \multirow[t]{15}{*}{ S. aureus } & Blank & 10 & $4.7 \times 10^{6} \pm 0.4$ & - \\
\hline & & 20 & $4.7 \times 10^{6} \pm 0.4$ & - \\
\hline & & 40 & $4.7 \times 10^{6} \pm 0.4$ & - \\
\hline & & 60 & $4.7 \times 10^{6} \pm 0.4$ & - \\
\hline & & 1440 & $2.5 \times 10^{6} \pm 0.8$ & - \\
\hline & Chitin (10 $\mathrm{mg} \mathrm{ml}^{-1}$ ) & 10 & $1.6 \times 10^{6} \pm 0.0$ & 7.0 \\
\hline & & 20 & $1.9 \times 10^{6} \pm 0.6$ & 6.0 \\
\hline & & 40 & $2.0 \times 10^{6} \pm 0.3$ & 5.5 \\
\hline & & 60 & $2.5 \times 10^{6} \pm 0.0$ & 4.0 \\
\hline & & 1440 & $4.9 \times 10^{6} \pm 0.3$ & 0 \\
\hline & Chitin betainate & 10 & $4.5 \times 10^{3} \pm 0.9$ & 45.2 \\
\hline & $\left(10 \mathrm{mg} \mathrm{ml}^{-1}\right)$ & 20 & $7.0 \times 10^{3} \pm 0.4$ & 42.3 \\
\hline & & 40 & $4.3 \times 10^{3} \pm 0.5$ & 45.5 \\
\hline & & 60 & $4.1 \times 10^{3} \pm 0.2$ & 45.8 \\
\hline & & 1440 & $2.5 \times 10^{1} \pm 3.5$ & 78.2 \\
\hline
\end{tabular}

Abbreviation: CFU, colony-forming units.

Data are shown as the mean \pm s.d. and are derived from three independent repeats.

coupling agent. DCC reacts with the carboxylic groups of CTA to form an $\mathrm{O}$-acylisourea intermediate, and then the hydroxyl groups of chitin and/or amino groups in partly deacetylated chitin react with
Table 2 The number of viable cells and percentage of reduction of the viable cell number of Escherichia coli and Staphylococcus aureus after exposure to different concentrations of the sample for $10 \mathrm{~min}$ for $E$. coli and $24 \mathrm{~h}$ for $S$. aureus compared with the control

\begin{tabular}{|c|c|c|c|c|}
\hline Microorganism & Sample & $\begin{array}{c}\text { Concentration } \\
\left(m g m l^{-1}\right)\end{array}$ & $\begin{array}{c}\text { Viable bacteria } \\
\left.\text { (CFU } \mathrm{ml}^{-1}\right)\end{array}$ & $\begin{array}{c}\% \\
\text { Reduction }\end{array}$ \\
\hline \multirow[t]{6}{*}{ E. coli } & Chitin & 10 & $3.0 \times 10^{6} \pm 0.0$ & - \\
\hline & & 5 & $1.9 \times 10^{6} \pm 0.1$ & - \\
\hline & & 1 & NA & NA \\
\hline & Chitin & 10 & $0.0 \pm 0.0$ & 100 \\
\hline & betainate & 5 & $1.0 \times 10^{6} \pm 0.1$ & 4.46 \\
\hline & & 1 & NA & NA \\
\hline \multirow[t]{6}{*}{ S. aureus } & Chitin & 10 & $2.5 \times 10^{6} \pm 0.8$ & - \\
\hline & & 5 & $3.1 \times 10^{6} \pm 0.8$ & - \\
\hline & & 1 & $5.0 \times 10^{6} \pm 0.1$ & - \\
\hline & Chitin & 10 & $2.5 \times 10^{1} \pm 3.5$ & 78.2 \\
\hline & betainate & 5 & $1.0 \times 10^{1} \pm 0.0$ & 84.6 \\
\hline & & 1 & $1.3 \times 10^{2} \pm 0.1$ & 68.5 \\
\hline
\end{tabular}

Abbreviations: CFU, colony-forming units; NA, not applicable.

Data are shown as the mean \pm s.d. and are derived from three independent repeats.

Table 3 Percentage of water absorption and the water contact angle of chitin, chitin betainate, chitin/PEG4000 and chitin betainate/ PEG4000 gels

\begin{tabular}{lcc}
\hline Samples & $\begin{array}{c}\text { Water absorption } \\
\text { (\% of dry weight) }\end{array}$ & $\begin{array}{c}\text { Contact ang/e } \\
\text { (degree) }\end{array}$ \\
\hline Chitin & $163 \pm 19$ & $53.6 \pm 1.7$ \\
Chitin betainate & $174 \pm 15$ & $34.0 \pm 2.0$ \\
Chitin/PEG4000 & $328 \pm 94$ & $44.4 \pm 1.7$ \\
Chitin betainate/PEG4000 & $435 \pm 23$ & $30.0 \pm 2.0$ \\
\hline
\end{tabular}

Abbreviation: PEG, polyethylene glycol.

Data are shown as the mean $\pm s . d$ and are derived from three independent repeats.

bData are shown as the mean \pm s.d. and are derived from five independent repeats.

the $\mathrm{O}$-acylisourea to yield chitin betainate and DCUrea as a byproduct. The chemical modification pathway is shown in Scheme 1.

Structural characterization of chitin betainate and its DS

Chitin, CTA and chitin betainate were structurally characterized by FTIR, as shown in Figure 1. Chitin exhibited characteristic FTIR peaks at $3500 \mathrm{~cm}^{-1}$ (O-H and N-H stretching), $2900 \mathrm{~cm}^{-1}$ (C-H stretching of methyl), $1650 \mathrm{~cm}^{-1}(\mathrm{C}=\mathrm{O}$ stretching, amide $\mathrm{I}), 1560 \mathrm{~cm}^{-1}(\mathrm{~N}-\mathrm{H}$ bending) and $1072 \mathrm{~cm}^{-1}$ (C-O stretching of pyranose ring). CTA exhibited the characteristic absorption of the carboxylic ester at $1726 \mathrm{~cm}^{-1}$ and of the C-N stretching at $1196 \mathrm{~cm}^{-1}$. Chitin betainate exhibited the same peaks as those of chitin and CTA but with the shift of the characteristic peak of the carboxylic ester of CTA at $1726 \mathrm{~cm}^{-1}$ to that of the ester linkage of chitin betainate at $1735 \mathrm{~cm}^{-1}$. This shift indicated that the quaternary ammonium groups (betainate) were successfully substituted onto chitin via acylation. Therefore, the DS of betainate was estimated from the degree of acylation using Equation (1) subtracted from that of the original chitin, revealing that betainate was highly substituted to chitin, with a DS of 1.70.

\section{Bactericidal activities}

If chitin betainate was to be used as a wound dressing, it must come into contact with infected wounds. We therefore evaluated its 


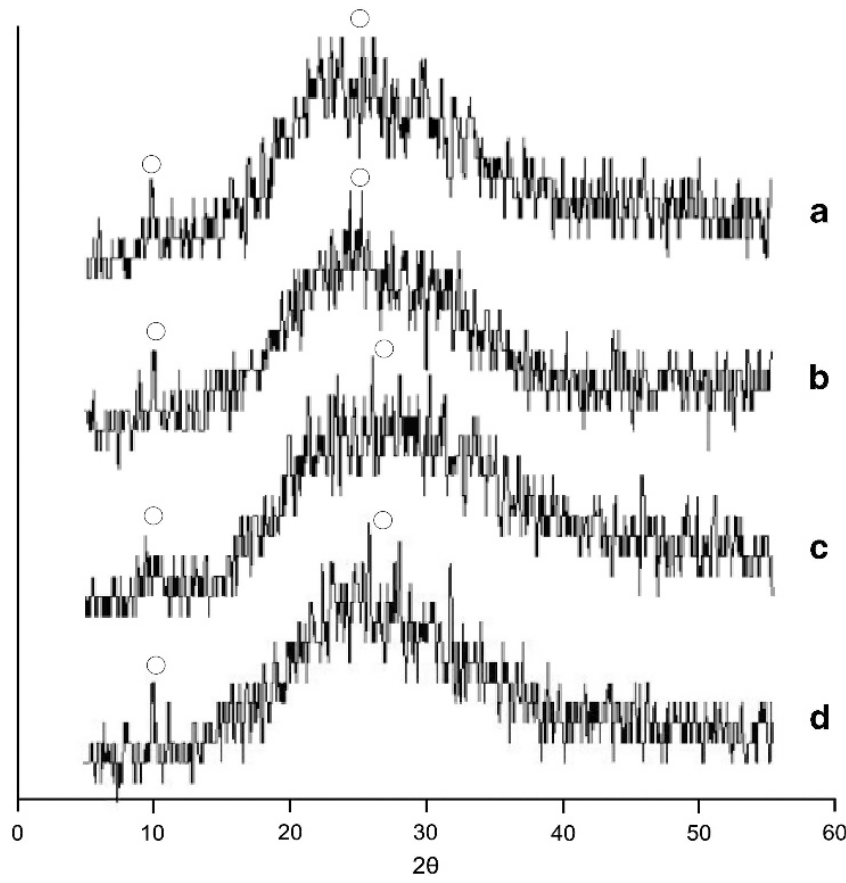

Figure 2 X-ray diffractograms of (a) chitin, (b) chitin/PEG4000, (c) chitin betainate and (d) chitin betainate/PEG4000 gels.

potential antibacterial activity in terms of the exposure time and the concentration dependence on the antibacterial activity.

With respect to the exposure time for bactericidal activity, Table 1 summarizes the total viable bacteria and the percentage of reduction of viable bacteria at a given time interval of exposure to each sample at a fixed concentration of $10 \mathrm{mg} \mathrm{ml}^{-1}$ compared with that of the control (same culture conditions but no sample added). We found that chitin did not exhibit significant antibacterial activity against either $S$. aureus (Gram-positive) or E. coli (Gram-negative). By contrast, chitin betainate completely killed all the E. coli within a 10-min exposure and induced $45.2-45.8 \%$ inhibition of $S$. aureus after exposure for $10-60 \mathrm{~min}$ and $78.2 \%$ inhibition after exposure for $24 \mathrm{~h}$. Thus, chitin betainate inhibited the growth of $E$. coli more efficiently than $S$. aureus over a short time. This behavior might be due to the differences in the bacterial cell wall. Gram-positive bacteria consisted of thicker peptidoglycan than did Gram-negative bacteria, which might be a barrier for the interaction between quaternary ammonium-positive groups and cytoplasmic membranes.

Jia et al. ${ }^{9}$ reported that quaternary ammonium with ethyl (C2) short alkyl substituent chain required less time to kill $E$. coli than S. aureus. Kim et al., ${ }^{16}$ however, reported that quaternary ammonium with octadecyl (C18) long alkyl substituent chain enhanced penetration through the thick layer of peptidoglycan and more efficiently killed $S$. aureus than it did E. coli.

With respect to the concentration dependence of the bactericidal activity, Table 2 summarizes the total viable bacteria and the percentage of reduction after exposure to the chitin betainate at a concentration of 10,5 and $1 \mathrm{mg} \mathrm{ml}^{-1}$ for $24 \mathrm{~h}$ for $S$. aureus and 10 min for E. coli, compared with that for chitin. As expected, chitin did not exhibit any significant antibacterial activity against either $S$. aureus or E. coli, whereas chitin betainate exhibited a concentration-dependent antibacterial activity against both $S$. aureus and E. coli.
In addition, chitin betainate more efficiently inhibited $E$. coli than $S$. aureus, despite the larger exposure time of $S$. aureus to the sample than of E. coli ( $24 \mathrm{~h}$ vs $10 \mathrm{~min}$ ).

In general, antibacterial action was usually achieved on contact with materials under the following sequence: adsorption onto the bacterial cell surface, diffusion through the cell wall, binding and disruption of the cytoplasmic membrane and the release of cytoplasmic substances, resulting in cell death. ${ }^{17}$ Two factors thought to be significant for antibacterial activity were the density of positive charges and the length of the substituent chain. The positive charge was thought to enhance the disruption of the cytoplasmic membrane or the outer membrane of lipopolysaccharide, whereas the long length of the hydrophobic substituent chain might enhance the penetration though peptidoglycan. The influence of quaternary ammonium structures on the efficiency of the selective bactericidal activities will be further investigated.

Chitin and chitin betainate hydrogels and their water absorption Chitin and chitin betainate gels were prepared in molds as described in the Materials and Methods section. The three-dimensional shape of chitin or chitin betainate gels could be easily stabilized by replacing the DMAc/5\% (w/v) LiCl solvent with a poor chitin solvent, such as distilled water and acetone. The water absorption of the gels was determined in triplicate, and the results are summarized in Table 3. Chitin gels had a water absorption of $163 \pm 19 \%$ (1.63 times its dried weight); the chitin betainate gels exhibited a slightly larger (1.07-fold) water absorption of $174 \pm 15 \%$. This difference may reflect the greater ability of the positively charged quaternary ammonium groups to induce hydrogen bonding with water molecules compared with the hydroxyl groups of the unmodified chitin. However, the water absorption of chitin or chitin betainate gels was enhanced by the formation of a semi-interpenetrating network with PEG4000. That is, when chitin or chitin betainate gels were prepared with $16.7 \%(\mathrm{w} / \mathrm{w})$ PEG4000, the chitin/PEG4000 gel exhibited a water absorption of $328 \pm 94 \%$, approximately twofold higher than that for the corresponding chitin gel. Moreover, the chitin betainate/PEG4000 gel exhibited even higher water absorption of $435 \pm 23 \%$, approximately 2.5-fold higher than the corresponding chitin betainate gel and 1.33-fold higher than the chitin/PEG4000 gel. This indicated that the interpenetration of chitin or chitin betainate with PEG4000 increased the hydrophilicity and thus enhanced water absorption.

\section{Hydrophilic-hydrophobic property}

The hydrophilic or hydrophobic property of the chitin, chitin betainate, chitin/PEG4000 and chitin betainate/PEG4000 gels was evaluated by measuring the water-contact angle. The lower the contact angle, the higher the hydrophilicity was, and vice versa. The contact-angle values of the different gels are summarized in Table 3. The chitin gels exhibited a contact angle of $53.6 \pm 1.7^{\circ}$; the chitin betainate gels had a lower contact angle, $34.0 \pm 2.0^{\circ}$. Thus, the chemical modification of chitin with the quaternary ammonium groups increased the hydrophilicity, which, as mentioned before, may be due to the positively charged quaternary ammonium groups inducing better hydrogen bonding with water molecules than the hydroxyl groups of chitin. In addition, the chitin/PEG4000 and chitin betainate/PEG4000 gels exhibited a lower water-contact angle than that of the corresponding chitin and chitin betainate gels, which exhibited values of approximately $44.4 \pm 1.7^{\circ}$ and $30.0 \pm 2.0^{\circ}$, respectively. This result indicated that the interpenetration of chitin or chitin betainate with PEG4000 increased the hydrophilicity or 
wettability, thus decreasing the water-contact angle and enhancing water absorption.

The addition of a small amount $(20 \%(w / w))$ of PEG6000 or PEG8000 to chitosan was previously reported to improve the wettability of the chitosan film surface. ${ }^{18-20}$ This enhanced hydrophilicity caused by the addition of either $\mathrm{M}_{\mathrm{W}}$ PEG was attributed to the immobilization of the PEG chains onto the material surface. ${ }^{18-20}$ The blending of the higher $M_{W}$ PEG8000 resulted in a lower wettability than that with the lower $\mathrm{M}_{\mathrm{W}}$ PEG6000. The result may also be partly attributed to the availability of the terminal hydroxyl groups of PEG, because the -OH groups may improve the hydrophilicity of materials. Therefore, the lower $\mathrm{M}_{\mathrm{W}}$ of PEG might further enhance hydrophilicity or wettability and water absorption.

\section{Crystallinity}

The crystallinity of the chitin, chitin betainate, chitin/PEG4000 and chitin betainate/PEG4000 gels were characterized by X-ray diffraction (Figure 2). The chitin gel exhibited $2 \theta$ peaks at $9.5^{\circ}$ and $24^{\circ}$, whereas the chitin betainate gel exhibited a sharp $2 \theta$ peak at $9.5^{\circ}$ and a broader $2 \theta$ peak at $28^{\circ}$. This shift in the $2 \theta$ peak and its broadening after quaternary ammonium modification indicated a lower $\mathrm{d}$-spacing in the crystalline region and a lower crystallinity level. $\alpha$-Chitin with high crystallinity mostly presented four sharp crystalline reflection peaks at $9.6^{\circ}, 19.6^{\circ}, 21.1^{\circ}$ and $23.7^{\circ} .{ }^{21}$ However, the chitin in this research was purified and was in the form of a gel in DMAc/LiCl that might interfere to the crystallinity; thus, the diffraction peaks may not be separately observed.

The chitin/PEG4000 gel exhibited essentially the same sharp $2 \theta$ peaks at $9.5^{\circ}$ and $24^{\circ}$ as those for chitin, indicating that the interpenetration of chitin with PEG4000 did not influence either the d-spacing of the chitin crystalline region or the amount of crystallinity. This may be because PEG4000 entangled over and around the crystalline regions or around the chitin chains in the amorphous regions.

The chitin betainate/PEG4000 gel exhibited $2 \theta$ peaks in the same locations $\left(9.5^{\circ}\right.$ and $\left.28^{\circ}\right)$ as those for the chitin betainate gel, but the $2 \theta$ peak at $28^{\circ}$ was narrower than that for the chitin betainate gel. Thus the interpenetration of chitin betainate with PEG4000 did not influence the d-spacing of the crystalline region but increased the level of crystallinity. This may be due to the positively charged quaternary ammonium groups of chitin betainate forming hydrogen bonds with the oxygen atoms of PEG, thereby increasing the crystallinity level.

Zhang et al. ${ }^{20}$ reported that chitosan modified with PEG-diacrylate on the amino groups of chitosan by Michael addition resulted in a decreased level of crystallinity. This effect indicated that the addition of PEG-diacrylate decreased the amount of amino groups and thus the amount of intermolecular hydrogen bonding, thereby decreasing the crystallinity level.

\section{CONCLUSION}

Chitin was successfully modified by acylation with positively charged quaternary ammonium betainate groups to a DS of 1.7 using DCC as the coupling agent. Whereas chitin did not exhibit any significant antibacterial activity against $S$. aureus and E. coli for levels up to $10 \mathrm{mg} \mathrm{ml}^{-1}$ and for exposures up to $24 \mathrm{~h}$, chitin betainate at $10 \mathrm{mg} \mathrm{ml}^{-1}$ exhibited complete bactericidal activity against E. coli within $10 \mathrm{~min}$ and $45.2 \%$ and $78 \%$ reduction against $S$. aureus after
$10 \mathrm{~min}$ and $24 \mathrm{~h}$, respectively. With respect to the efficiency of the minimal killing concentration, chitin betainate exhibited a concentration-dependent bactericidal activity against both $S$. aureus and E. coli but was more efficient against $E$. coli than against $S$. aureus. Chitin betainate gels had a higher ability to absorb water than chitin gels, reflecting their greater hydrophilic property. The addition of $16.7 \%$ $(\mathrm{w} / \mathrm{w})$ PEG4000 increased the water adsorption of chitin and chitin betainate by 2.0 - and 2.5 -fold, respectively.

\section{ACKNOWLEDGEMENTS}

This research was financially supported by A1B1-MICO (TRF) and the C.U. Graduate School Thesis Grant, Chulalongkorn University. We greatly appreciate the Integrated Innovation Academic Center (IIAC), Chulalongkorn University Centenary Academic Development Project for partial financial support.

1 Usami, Y., Okamoto, Y., Takayama, T., Shigemasa, Y. \& Minami, S. Chitin and chitosan stimulate canine polymorphonuclear cells to release leukotriene $\mathrm{B}_{4}$ and prostaglandina E2. J. Biomed. Mater. Res. 42, 517-522 (1998).

2 Shigemasa, Y., Oota, H., Tokura, S., Matsuhashi, A. \& Minami, S. Biological filling agent and wound healing agent. EP Patent No. 0477979 (1992).

3 Okamoto, Y., Minami, S., Matsuhashi, A., Sashiwa, H., Shigemasa, Y., Tanigawa, T. Tanaka, Y. \& Tokura, S. Polymeric $N$-acytyl-D-glucosamine (chitin) induces histionic activation in dogs. J. Vet. Med. Sci. 55, 739-742 (1993).

4 Okamoto, Y., Minami, S., Matsuhashi, A., Sashiwa, H., Saimoto, H., Shigemasa, Y., Tanigawa, T., Tanaka, Y. \& Tokura, S. Application of polymeric $\mathrm{N}$-acetyl-D-glucosamine (chitin) to veterinary practice. J. Vet. Med. Sci. 55, 743-747 (1993).

5 Okamoto, Y., Shibazaki, K., Minami, S., Matsuhashi, A., Tanioka, S. \& Shigemasa, Y. Evaluation of chitin and chitosan on open wound healing in dogs. J. Vet. Med. Sci. $\mathbf{5 7}$ 851-854 (1995)

6 Okamoto, Y., Southwood, L., Stashak, T. S., Norrdin, R. W., Nelson, A. W., Minami, S., Matsuhashi, A., Kato, K. \& Shigemasa, Y. Effect of chitin on non-woven fabric implant in tendon healing. Carbohydr. Polym. 33, 33-38 (1997).

7 Okamoto, Y., Kawakami, K., Miyatake, K., Morimoto, M., Shigemasa, Y. \& Minami, S Analgesic effects of chitin and chitosan. Carbohydr. Polym. 49, 249-252 (2002).

8 Tanodekaew, S., Prasitsilp, M., Swasdison, S., Thavornyutikarn, B., Pothsree, T. \& Pateepasen, R. Preparation of acrylic grafted chitin for wound dressing application. Biomaterials 25, 1453-1460 (2004).

$9 \mathrm{Jia}, \mathrm{Z}$., Shen, D. \& Xu, W. Synthesis and antibacterial activities of quaternary ammonium salt of chitosan. Carbohydr. Res. 333, 1-6 (2001).

10 Qin, C., Xiao, Q., Li, H., Fang, M., Liu, Y., Chen, X. \& Li, Q. Calorimetric Studies of the action of chitosan- $N$-2-hydroxypropyl trimethyl ammonium chloride on the growth of microorganisms. Int. J. Biol. Macromol. 34, 121-126 (2004).

$11 \mathrm{Liu}, \mathrm{H}$., Du, Y., Yang, J. \& Zhu, H. Structural characterization and antimicrobial activity of chitosan/betaine derivative complex. Carbohydr. Polym. 55, 291-297 (2004).

12 Holappa, J., Hjálmarsdóttir, M., Másson, M., Rúnarsson, Ő., Asplund, T., Soininen, P. Nevalainen, T. \& Järvinen, T. Antimicrobial activity of chitosan $\mathrm{N}$-betainates. Carbohydr. Polym. 65, 114-118 (2006).

$13 \mathrm{Lim}, \mathrm{S}$. H. \& Hudson, S. M. Synthesis and antimicrobial activity of a water-soluble chitosan derivative with a fiber-reactive group. Carbohydr. Res. 339, 313-319 (2004).

$14 \mathrm{He}$, G., Wang, Z., Zheng, H., Yin, Y., Xiong, X. \& Lin, R. Preparation, characterization and properties of aminoethyl chitin hydrogels. Carbohydr. Polym. 90, 1614-1619 (2012).

15 Kim, J. Y., Lee, J. K., Lee, T. S. \& Park, W. H. Synthesis of chitooligosaccharide derivative with quaternary ammonium group and its antimicrobial activity against Streptococcus mutans. Int. J. Biol. Macromol. 32, 23-27 (2003).

16 Kim, Y. S., Kim, H. W., Lee, S. H., Shin, K. S., Hur, H. W. \& Rhee, Y. H. Preparation of alginate-quaternary ammonium complex beads and evaluation of their antimicrobia activity. Int. J. Biol. Macromol. 41, 36-41 (2007).

17 Massi, L., Guittard, F., Géribaldi, S., Levy, R. \& Duccini, Y. Antimicrobial properties of highly fluorinated bis-ammonium salts. Int. J. Antimicrob. Agents 21, 20-26 (2003).

18 Zhang, M., Li, X. H., Gong., Y. D., Zhao, N. M. \& Zhang, X. F. Properties and biocompatibility of chitosan films modified by blending with PEG. Biomaterials 23 , 2641-2648 (2002).

19 Ikada, Y. Surface modification of polymers for medical applications. Biomaterials 15 725-736 (1994)

20 Zhang, X., Yang, D. \& Nie, J. Chitosan/polyethylene glycol diacrylate films as potential wound dressing material. Int. J. Biol. Macromol. 43, 456-462 (2008).

21 Jang, M., Kong, B., Jeong, Y., Lee, C. H. \& Nah, J. Physicochemical characterization of $\alpha$-chitin, $\beta$-chitin, and $\gamma$-chitin separated from natural resources. J. Polym. Sci. Part A Polym. Chem. 42, 3423-3432 (2004). 